\title{
Prediction of Cobb-angle for Monitoring System in Adolescent Girls with Idiopathic Scoliosis using Multiple Regression Analysis
}

\author{
Eun Ji Seo, Ahnryul Choi, Seung Eel Oh, Hyun Joon Park, Dong Jun Lee, Joung H. Mun* \\ Department of Bio-Mechatronic Engineering, College of Biotechnology \& Bioengineering, \\ Sungkyunkwan University, 300 Chunchun, Jangan, Suwon, Gyeonggi, Korea
}

Received: October $18^{\text {th }}, 2012$; Revised: February $28^{\text {th }}, 2013$; Accepted: February $28^{\text {th }}, 2013$

\section{Abstract}

Purpose: The purpose of this study was to select standing posture parameters that have a significant difference according to the severity of spinal deformity, and to develop a novel Cobb angle prediction model for adolescent girls with idiopathic scoliosis. Methods: Five normal adolescents girls with no history of musculoskeletal disorders, 13 mild scoliosis patients (Cobb angle: $10^{\circ}-25^{\circ}$ ), and 14 severe scoliosis patients (Cobb angle: $25^{\circ}-50^{\circ}$ ) participated in this study. Six infrared cameras (VICON) were used to acquire data and 35 standing parameters of scoliosis patients were extracted from previous studies. Using the ANOVA and post-hoc test, parameters that had significant differences were extracted. In addition, these standing posture parameters were utilized to develop a Cobb-angle prediction model through multiple regression analysis. Results: Twenty two of the parameters showed differences between at least two of the three groups and these parameters were used to develop the multi-linear regression model. This model showed a good agreement $\left(\mathrm{R}^{2}=0.92\right)$ between the predicted and the measured Cobb angle. Also, a blind study was performed using 5 random datasets that had not been used in the model and the errors were approximately $3.2 \pm 1.8$. Conclusions: In this study, we demonstrated the possibility of clinically predicting the Cobb angle using a non-invasive technique. Also, monitoring changes in patients with a progressive disease, such as scoliosis, will make possible to have determine the appropriate treatment and rehabilitation strategies without the need for radiation exposure.

Keywords: Cobb angle, Idiopathic scoliosis, Motion capture, Multiple regression analysis, Static posture

\section{Introduction}

The prevalence of scoliosis is approximately $2-3 \%$ in adolescents between $10-16$ years worldwide and during the growth of the skeletal system, especially, the angle of curvature progresses annually (Duval et al., 1971). Also, it has been reported that growth of the spine and an increase in the curvature are positively correlated (Ylikoski, 1993) and $14.7 \%$ of scoliosis patients showed an annual increase in disease (Soucacos et al., 1998). Therefore, the goal of scoliosis treatment is to prevent the

\footnotetext{
* Corresponding author: Joung H. Mun

Tel: +82-31-290-7827; Fax: +82-31-290-7820

E-mail: jmun@skku.edu
}

progression of the disease through regular observations (Fortin et al., 2010, 2011).

Medical determinations on the severity of scoliosis is often based on the Cobb angle (Cobb, 1948), which is a measure of the degree of curvature of the spine on the coronal plane based on x-ray analysis. However, there are many issues associated with radiation diagnostics and this method cannot measure secondary deformations on the pelvis and lower limb, since only information on the deformation of the spine can be obtained from radiography images. Therefore several non-invasive methods of diagnosing scoliosis have been examined as alternatives to radiation based diagnosis (Fortin et al. 2011; Sapkas et al., 2003; Jaremko et al, 2002). 
Seo et al. Prediction of Cobb-angle for Monitoring System in Adolescent Girls with Idiopathic Scoliosis using ... Journal of Biosystems Engineering • Vol. 38, No. 1, 2013 • www.jbeng.org

Representative non-invasive monitoring methods include palpation techniques by experts, measurements using a scoliometer and a 3D image reconstruction device. Palpation is a simple method of determining the degree of severity based on clinician or physical therapist's sense of touch of the curvature, but it has limitations in regards to objectivity, consistency and detailed diagnosis (Fortin et al., 2011). A scoliometer is a simple and reliable piece of equipment, but It provides only one parameter related to the posture and has a low degree of precision (Masso and Gorton, 2000; Sapkas et al., 2003). In addition, this approach can only be used to determine whether scoliosis occurred or not. The 3D imaging device utilizes a 3D scanner that can image the 3D shape of the body segment surface including the spine (Poncet et al., 2000). Using the scanner, Jaremko et al. (2002) identified trunk asymmetry parameters and developed a Cobb angle prediction model, but the Cobb angle prediction rate of this approach, within $5^{\circ}$ of error, was as low as $64 \%$. Many methods to non-invasively predict the Cobb angle have been developed (Jaremko et al., 2002; Korovessis and Stamatakis, 1996; Sapkas et al., 2003); however, there have been a number of limitations to these methods in regards to the degree of accuracy for actual periodic observations.

Scoliosis accompanies three-dimensional deformity with rotation of the spine in the transverse plane (Stokes et al., $1988 ; 1989$ ) and can cause many secondary deformations. Zhu et al. (2011) showed deformation of the spine in scoliosis-patients accompanies deformation in the rib length; Stylianides et al. (2012) showed that pelvic orientation and geometry of adolescent idiopathic scoliosis girls are deformed in standing posture. Also, Dalleau et al. (2012) showed the differences in variables of pelvis morphology and trunk posture in a static state accordance to the severities of the scoliosis patients, moderate and sever; Zabjek et al. (2008) evaluated the postural characteristics of idiopathic scoliosis patients with different types of spinal curvature. In terms of biomechanics, these deformations can be led to postural unbalance and have a negative effect on human activities (Fortin et al., 2010).

As shown in the results of previous studies, the existing studies have done related to the Cobb angle did not reflect the deformation parameters even the scoliosis causes overall posture deformations. For an accurate scoliosis diagnosis, the Cobb angle should be predicted with the three-dimensional deformations from a biomechanics point of view. In this study, we developed a prediction

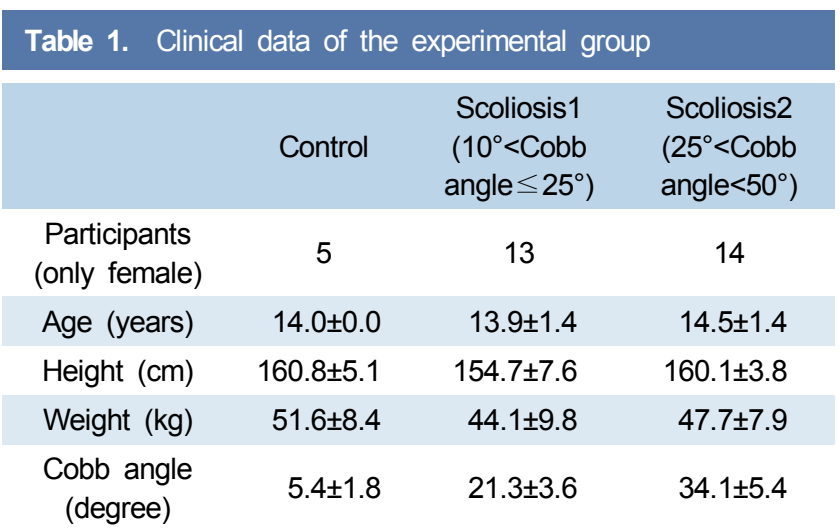

Values are presented as mean $\pm S D$.

model using standing posture parameters from the whole human body and the accuracy of predicting the Cobb angle in patients was assessed.

\section{Materials and Methods}

\section{Subjects \& apparatus}

All experiments were performed after approval by the Institutional Review Board (IRB). The subjects included 27 adolescent girls with idiopathic scoliosis those have no history of spinal surgery and 5 healthy adolescent girls with no history of spine-related diseases. The 5 normal subjects had a Cobb angle of less than $10^{\circ}$ (Reamy and Slakey, 2001), and the patients were divided into 2 groups: Scoliosis1 (Cobb-angle: $10^{\circ}-25^{\circ}$ ) which was the mild case group (Chow et al., 2006), and Scoliosis2 (Cobb-angle: $25^{\circ}-50^{\circ}$ ), which was the severe group. Patients with a Cobb angle of more than $50^{\circ}$ were excluded from this study because this degree of scoliosis requires surgery (Karol et al., 2001). For the analysis, 6 infrared cameras (VICON, Oxford Metrics, Oxford, UK) were used, and each camera was synchronized using the Vicon 460 system. The acquired 2D coordinates of the markers from each synchronized camera were reconstructed into 3D coordinates using the Workstation program, and each coordinate acquired during a period of time was calculated into a value for each posture using an averaging process. Table 1 shows clinical information of the subjects that participated in the study.

\section{Experimental protocol}

A total of 37 infrared optical markers were attached to the subjects in order to extract static standing posture 

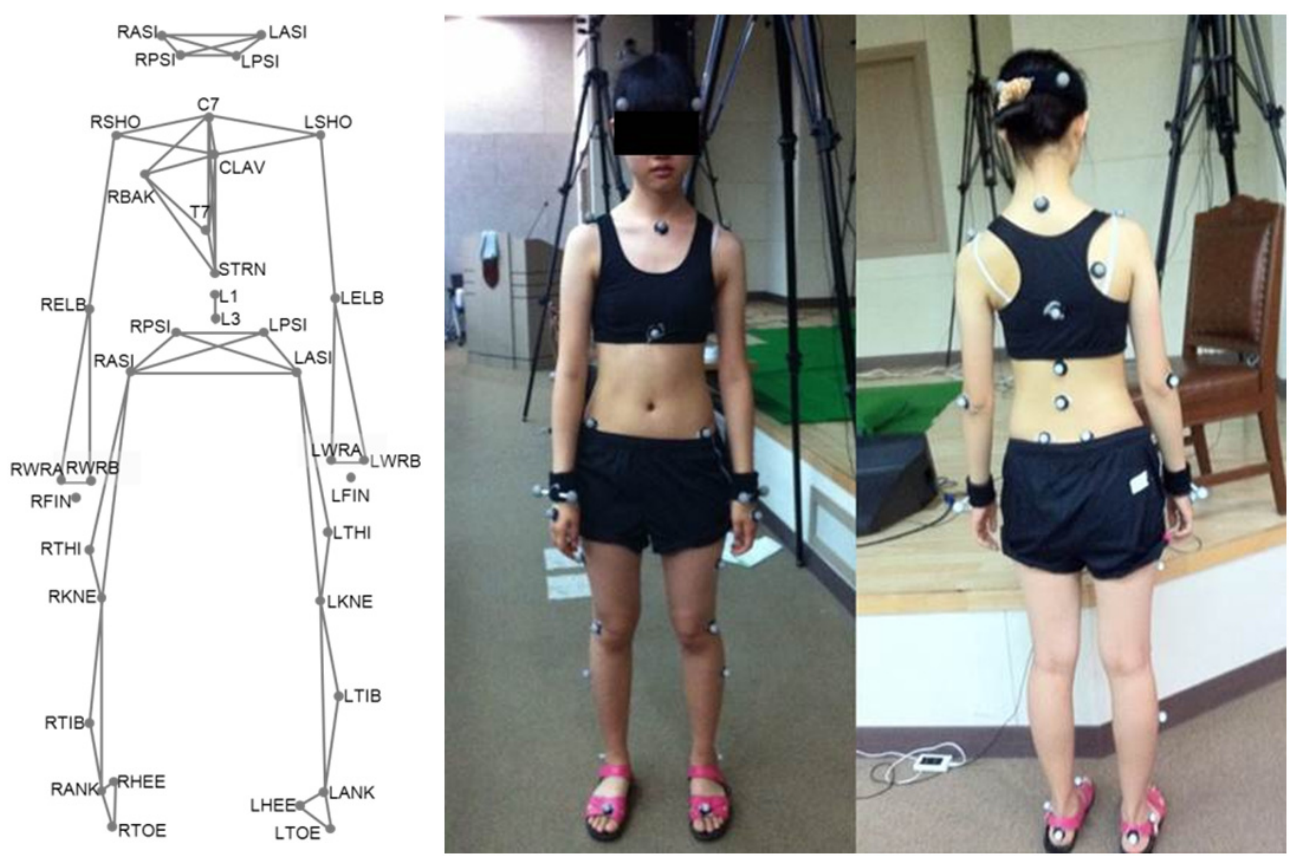

Figure 1. Locations of the attached optical sensors.

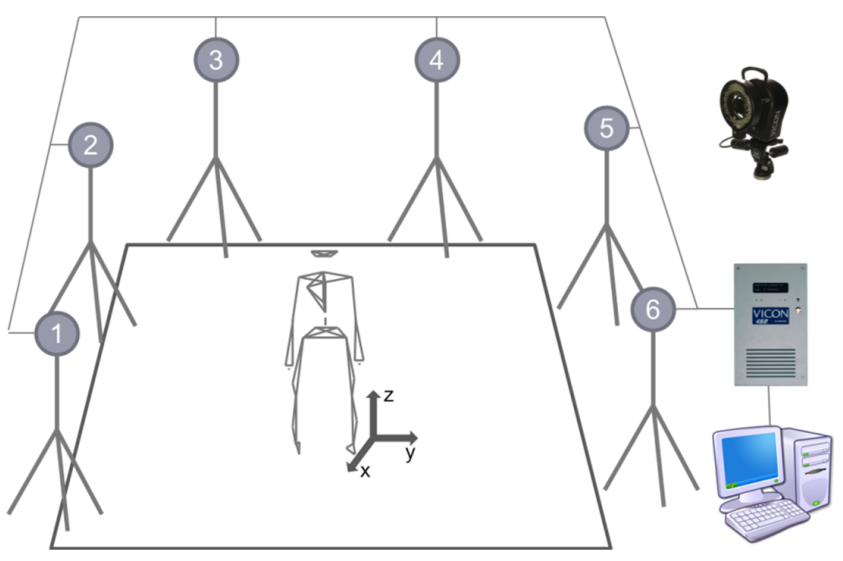

Figure 2. Experimental environment.

parameters. The location of the attached markers was based on the modified Helen-Hayes markerset (Davis et al., 1991), and additional markers were attached on the 1st and 3rd lumbar vertebra to measure the parameters related to spinal deformity. Figure 1 shows the location of the optical markers that were attached to the subjects.

The subjects were asked to spread their legs shoulder width apart, lower both arms and stare in front of themselves for 5 seconds. For the upright posture, subjects were asked to maintain a posture that felt comfortable. The posture was captured three times repeatedly, and the data collected was used in the analysis. Also, a global coordinate system was defined; anterior/posterior dir- ection of the subject was the $\mathrm{x}$-axis, medial/lateral direction was the $y$-axis, and the up/down direction was the z-axis. The overall experimental environments are shown in the Figure 2.

\section{Parameters from literature review}

In this study, the posture parameters described in previous studies (Fortin et al., 2012, 2010; Negrini et al., 2003; Nault et al., 2002; Masso and Gorton, 2000; Byl and Gray., 1993) were used for standing posture analysis of patients with idiopathic scoliosis. A total of 35 standing posture parameters were selected (Table 2).

\section{Statistical analysis}

Within the scoliosis patients' standing posture parameters, which were selected based on previous studies, ANOVA and post-hoc tests were performed to determine which parameters were related to the severity of disease. ANOVA is a statistical method to determine whether there are differences between groups. However, ANOVA does not show in which groups the differences occur. Thus, Tukey method was applied for a post-hoc test.

After selecting the posture parameters that displayed differences in terms of the severity of scoliosis, multi regression analysis was performed for Cobb-angle prediction model build. The general equation used for the multi regression analysis is shown below. 
Seo et al. Prediction of Cobb-angle for Monitoring System in Adolescent Girls with Idiopathic Scoliosis using ... Journal of Biosystems Engineering • Vol. 38, No. 1, $2013 \cdot$ www.jbeng.org

Table 2. Parameters from literature review

\begin{tabular}{|c|c|c|c|}
\hline No & Parameters & Description & Unit \\
\hline 1 & Sho-Pel aligned $(x)^{a)}$ & Shoulder aligned with respect to the pelvis in transverse plane & deg \\
\hline 2 & Sho-Pel aligned(y) & Shoulder aligned with respect to the pelvis in frontal plane & deg \\
\hline 3 & Head-Pel aligned $(x)^{b)}$ & Head aligned with respect to the pelvis in transverse plane & deg \\
\hline 4 & Head-Pel aligned $(y)^{b)}$ & Head aligned with respect to the pelvis in frontal plane & deg \\
\hline 5 & Ratio of left-right ${ }^{c)}$ & The ratio of left and right shoulder to foot aligned & - \\
\hline 6 & Diff leg length ${ }^{c}$ & Leg length difference & $\mathrm{mm}$ \\
\hline 7 & Trunk list ${ }^{d)}$ & Vertical distance between $\mathrm{C} 7$ and PSI, center of LPSI and RPSI & $\mathrm{mm}$ \\
\hline 8 & $\mathrm{C} 7-\mathrm{T} 10^{\mathrm{d})}$ & Tilted angle from $\mathrm{C} 7$ to $\mathrm{T} 7$ by vertical line from $\mathrm{C} 7$ & $\operatorname{deg}$ \\
\hline 9 & T7-L1 ${ }^{\mathrm{d})}$ & Tilted angle from $\mathrm{T} 10$ to $\mathrm{L} 1$ by vertical line from $\mathrm{C} 7$ & deg \\
\hline 10 & L1-L3 ${ }^{d)}$ & Tilted angle from L1 to L3 by vertical line from $\mathrm{C} 7$ & deg \\
\hline 11 & L3-PSI $^{\text {d) }}$ & Tilted angle from L1 to PSI by vertical line from C7 & deg \\
\hline 12 & C7-T10-L1 ${ }^{\mathrm{e})}$ & Angle between $\mathrm{C} 7, \mathrm{~T} 10$ and L1 & deg \\
\hline 13 & Shoulder tilt ${ }^{a}$ & Shoulders aligned in transverse plane & $\operatorname{deg}$ \\
\hline 14 & Shoulder elevation ${ }^{\text {a) }}$ & Shoulder aligned in frontal plane & deg \\
\hline 15 & Pelvis tilt ${ }^{b)}$ & Pelvis aligned in transverse plane & deg \\
\hline 16 & Pelvis obliquity ${ }^{\text {b) }}$ & Pelvis aligned in frontal plane & $\operatorname{deg}$ \\
\hline 17 & Pelvis rotation angle ${ }^{\mathrm{c})}$ & Pelvis aligned in sagittal plane & deg \\
\hline 18 & Trunk angle $(x)^{c)}$ & Trunk aligned in transverse plane & deg \\
\hline 19 & Trunk angle $(\mathrm{y})^{\mathrm{c})}$ & Trunk aligned in frontal plane & deg \\
\hline 20 & Trunk angle $\left.(z)^{c}\right)$ & Trunk aligned in sagittal plane & deg \\
\hline 21 & Head angle $(x)^{c)}$ & Head aligned in transverse plane & deg \\
\hline 22 & Head angle $(y)^{c)}$ & Head aligned in frontal plane & deg \\
\hline 23 & Head angle $(z)^{c)}$ & Head aligned in sagittal plane & deg \\
\hline 24 & Knee joint angle $L(x)^{c)}$ & Left knee joint angle in transverse plane & deg \\
\hline 25 & Knee joint angle $\mathrm{L}(\mathrm{y})^{\mathrm{c})}$ & Left knee joint angle in frontal plane & deg \\
\hline 26 & Knee joint angle $L(z)^{c)}$ & Left knee joint angle in sagittal plane & deg \\
\hline 27 & Knee joint angle $R(x)^{c)}$ & Right knee joint angle in transverse plane & deg \\
\hline 28 & Knee joint angle $R(y)^{c)}$ & Right knee joint angle in frontal plane & deg \\
\hline 29 & Knee joint angle $R(z)^{c)}$ & Right knee joint angle in sagittal plane & deg \\
\hline 30 & Hip joint angle $L(x)^{c)}$ & Left hip joint angle in transverse plane & deg \\
\hline 31 & Hip joint angle $\mathrm{L}(\mathrm{y})^{\mathrm{c})}$ & Left hip joint angle in frontal plane & deg \\
\hline 32 & Hip joint angle $L(z)^{c)}$ & Left hip joint angle in sagittal plane & deg \\
\hline 33 & Hip joint angle $R(x)^{c)}$ & Right hip joint angle in transverse plane & deg \\
\hline 34 & Hip joint angle $R(y)^{c)}$ & Right hip joint angle in frontal plane & deg \\
\hline 35 & Hip joint angle $R(z)^{c)}$ & Right hip joint angle in sagittal plane & deg \\
\hline
\end{tabular}

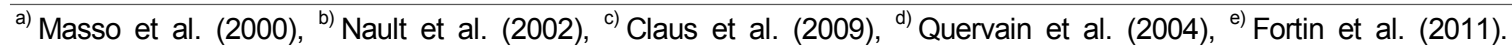

$$
Y=a_{0}+a_{1} X_{1}+a_{2} X_{2}+\cdots+a_{n} X_{n}
$$

The Cobb angle was the independent variable $Y$, the selected standing posture parameters of scoliosis patients were the dependent variables $X_{1}, X_{2}, \cdots, X_{n}$, the linear coefficients $a_{0}, a_{1}, \cdots, a_{n}$ were used for predictions and the number of applied posture parameters corresponds to $n$. Among the 32 subjects, 27 were used in the multi regression analysis prediction model for Cobb angle prediction. The forward selection method was used for the variable selection. SAS Window V9.1 (SAS Institute Inc., Cary, NC, USA) was used as the statistical analysis program 
Seo et al. Prediction of Cobb-angle for Monitoring System in Adolescent Girls with Idiopathic Scoliosis using ... Journal of Biosystems Engineering • Vol. 38, No. 1, 2013•www.jbeng.org

Table 3. ANOVA and post-hoc test results

\begin{tabular}{|c|c|c|c|c|c|}
\hline No & Parameter & Normal & Scoliosis1 & Scoliosis2 & $P$-value \\
\hline 1 & Sho-Pel aligned(x) & $0.922 \pm 0.944^{1}$ & $3.021 \pm 2.143^{1,2}$ & $3.676 \pm 1.877^{2}$ & 0.032 \\
\hline 2 & Sho-Pel aligned(y) $)^{b)}$ & $1.628 \pm 2.157^{1}$ & $1.145 \pm 0.774^{1}$ & $2.153 \pm 1.553^{2}$ & 0.195 \\
\hline 4 & Head-Pel aligned $(y)^{a)}$ & $5.506 \pm 5.383^{1}$ & $2.460 \pm 2.682^{2}$ & $3.611 \pm 2.554^{1,2}$ & 0.195 \\
\hline 5 & Ratio of left-right ${ }^{a)}$ & $0.069 \pm 0.074^{1}$ & $0.343 \pm 0.347^{2}$ & $0.409 \pm 0.286^{2}$ & 0.102 \\
\hline 6 & Diff Leg Length ${ }^{\mathrm{b})}$ & $0.004 \pm 0.001^{1}$ & $0.007 \pm 0.005^{1}$ & $0.011 \pm 0.007^{1,2}$ & 0.038 \\
\hline 7 & Trunk List ${ }^{a)}$ & $2.480 \pm 1.801^{1}$ & $12.047 \pm 10.516^{2}$ & $7.568 \pm 7.418^{1,2}$ & 0.098 \\
\hline 9 & $\mathrm{~T} 7-\mathrm{L} 1^{\mathrm{b})}$ & $1.197 \pm 0.478^{1}$ & $1.955 \pm 2.263^{1}$ & $3.980 \pm 3.039^{2}$ & 0.052 \\
\hline 12 & C7-T7-L1 ${ }^{\text {a) }}$ & $4.203 \pm 2.730^{1}$ & $13.239 \pm 15.909^{1,2}$ & $20.605 \pm 17.159^{2}$ & 0.125 \\
\hline 13 & Shoulder tilt ${ }^{\mathrm{a})}$ & $1.150 \pm 0.361^{1}$ & $4.539 \pm 3.981^{2}$ & $3.885 \pm 2.943^{2}$ & 0.152 \\
\hline 15 & Pelvis tilt ${ }^{\mathrm{a})}$ & $3.817 \pm 2.469^{1}$ & $11.171 \pm 9.734^{2}$ & $10.141 \pm 6.532^{2}$ & 0.197 \\
\hline 19 & Trunk angle $(y)^{a}$ & $1.093 \pm 0.471^{1}$ & $4.361 \pm 4.316^{2}$ & $3.271 \pm 2.349^{2}$ & 0.168 \\
\hline 23 & Head angle $(z)^{a)}$ & $1.870 \pm 0.387^{1}$ & $6.600 \pm 5.138^{2}$ & $6.195 \pm 4.214^{2}$ & 0.117 \\
\hline 24 & Knee joint angle $L(x)^{a)}$ & $3.301 \pm 2.949^{1}$ & $7.439 \pm 6.036^{2}$ & $8.418 \pm 5.044^{2}$ & 0.190 \\
\hline 25 & Knee joint angle $L(y)^{c}$ & $0.766 \pm 0.430^{1}$ & $4.698 \pm 2.289^{2}$ & $3.266 \pm 2.047^{3}$ & 0.003 \\
\hline 26 & Knee joint angle $L(z)^{a)}$ & $6.065 \pm 3.481^{1}$ & $14.697 \pm 9.626^{2}$ & $17.412 \pm 11.433^{2}$ & 0.108 \\
\hline 27 & Knee joint angle $R(x)^{a)}$ & $2.256 \pm 1.121^{1}$ & $7.025 \pm 4.515^{2}$ & $7.914 \pm 5.833^{2}$ & 0.097 \\
\hline 28 & Knee joint angle $R(y)^{a)}$ & $1.636 \pm 1.141^{1}$ & $3.304 \pm 2.448^{2}$ & $2.188 \pm 1.408^{1,2}$ & 0.170 \\
\hline 30 & Hip joint angle $L(x)^{a)}$ & $6.375 \pm 3.748^{1}$ & $10.758 \pm 6.569^{2}$ & $6.745 \pm 4.263^{1,2}$ & 0.114 \\
\hline 31 & Hip joint angle $L(y)^{a)}$ & $6.156 \pm 3.150^{1}$ & $16.016 \pm 8.244^{2}$ & $14.234 \pm 8.985^{2}$ & 0.082 \\
\hline 34 & Hip joint angle $R(y)^{a)}$ & $2.783 \pm 1.034^{1}$ & $6.058 \pm 2.965^{2}$ & $4.439 \pm 3.409^{1,2}$ & 0.111 \\
\hline 35 & Hip joint angle $R(z)^{a)}$ & $4.026 \pm 1.775^{1}$ & $13.878 \pm 13.701^{2}$ & $13.533 \pm 8.206^{2}$ & 0.179 \\
\hline
\end{tabular}

a) Significantly different parameter between normal and patients(Scoliosis1, Scoliosis2) group.

b) Significantly different parameter between mild(normal, Scoliosis1) and severe patients group.

c) Significantly different parameter between normal, Scoliosis1 and Scoliosis2 group.

${ }^{1 \sim 3}$ Same number labeled represents the same subject group.

\section{Results and Discussion}

Parameters that were found to be related with the severity of scoliosis disease were selected using the ANOVA and post-hoc test. The significance level for selection was set to less than 0.2 , which was chosen based on a previous study by Fisher et al. (1991). Table 3 shows the variables that were significantly different in the ANOVA and post-hoc test.

21 parameters were found to be relation with the severity of scoliosis disease. The results from the post-hoc test for these 21 parameters were as follows. Differences between the normal patients and the scoliosis were observed for 18 of the variables, no differences between the normal and mild scoliosis patients but differences between normal and severe patients were observed for 3 of the variables and differences between both normal and mild patients (Scoliosis1) and severe patients (Scoliosis2) were observed for one of the variables.

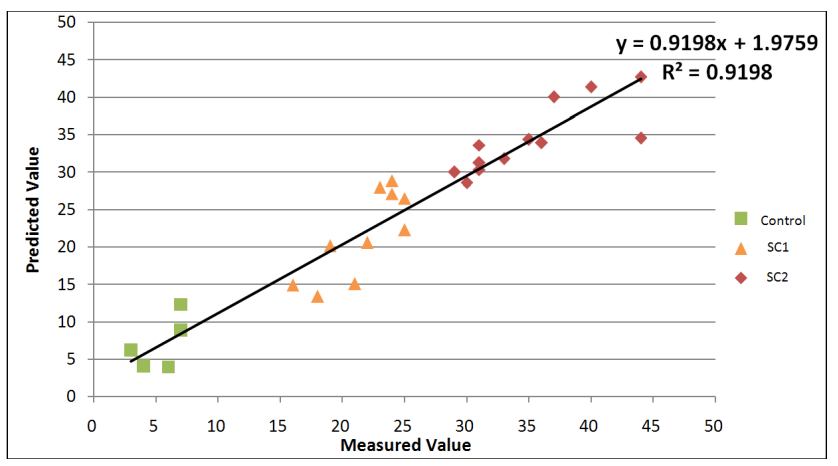

Figure 3. Multiple linear regression model for Cobb-angle prediction.

These 21 parameters were used to develop a Cobb angle prediction model. Among the 32 data sets, 27 data sets were used to develop the model. The forward selection method was used to select the parameters for generation of the regression models. Using this approach a Cobb angle prediction model with high accountability between the predicted and the measured values, $R^{2}=0.9198$, 
was generated. The linear equation for the measured $(X)$ and predicted $(\mathrm{Y})$ can be represented by $\mathrm{Y}=0.9198 \mathrm{X}+$ 1.9759, and the regression line are shown in Figure 3. The explanations for the posture parameters are shown in Table 4.

The linear equation for the measured $(\mathrm{X})$ and predicted $(\mathrm{Y})$ can be represented by $\mathrm{Y}=0.9198 \mathrm{X}+1.9759$, and the model had a high accuracy of $R^{2}=0.9198$. The slope of the regression equation 0.9198 from the prediction model using least square estimation, which is close to 1 , means high correlation between the predicted value and the measured value. However, still the prediction model has a little offset 1.9759 by the $y$-intercept, and it seems to be related with the not sufficient number of the subjects.

To validate the Cobb angle prediction model, the data from 5 arbitrary patients not utilized in model develop-

\section{Table 4. Multiple linear regression analysis Result}

\begin{tabular}{lccc}
\multicolumn{1}{c}{ Parameters } & $\begin{array}{c}\text { Regression } \\
\text { coefficient }\end{array}$ & $\begin{array}{c}\text { Standard } \\
\text { estimate Error }\end{array}$ & $\mathrm{Pr}<\mathrm{F}$ \\
\hline Intercept & -2.944 & 3.608 & 0.430 \\
\hline Sho-Pel aligned(x) & 2.194 & 0.804 & 0.018 \\
\hline Ratio of left-right & -6.628 & 4.163 & 0.137 \\
\hline T7-L1 & 1.005 & 0.366 & 0.018 \\
\hline C7-T7-L1 & 0.300 & 0.087 & 0.005 \\
\hline Shoulder tilt & -0.472 & 0.434 & 0.298 \\
\hline Trunk angle(y) & 0.306 & 0.325 & 0.366 \\
\hline Head angle(z) & 0.073 & 0.025 & 0.014 \\
\hline Knee joint angle L(x) & 0.828 & 0.309 & 0.020 \\
\hline Knee joint angle L(z) & 0.325 & 0.146 & 0.046 \\
\hline Knee joint angle $\mathrm{R}(\mathrm{x})$ & 0.668 & 0.378 & 0.103 \\
\hline Knee joint angle $\mathrm{R}(\mathrm{y})$ & -1.328 & 0.820 & 0.131 \\
\hline Hip joint angle $\mathrm{L}(\mathrm{x})$ & -0.716 & 0.251 & 0.015 \\
\hline Hip joint angle $\mathrm{L}(\mathrm{z})$ & 0.589 & 0.164 & 0.004 \\
\hline Hip joint angle $\mathrm{R}(\mathrm{z})$ & 0.122 & 0.099 & 0.243 \\
\hline
\end{tabular}

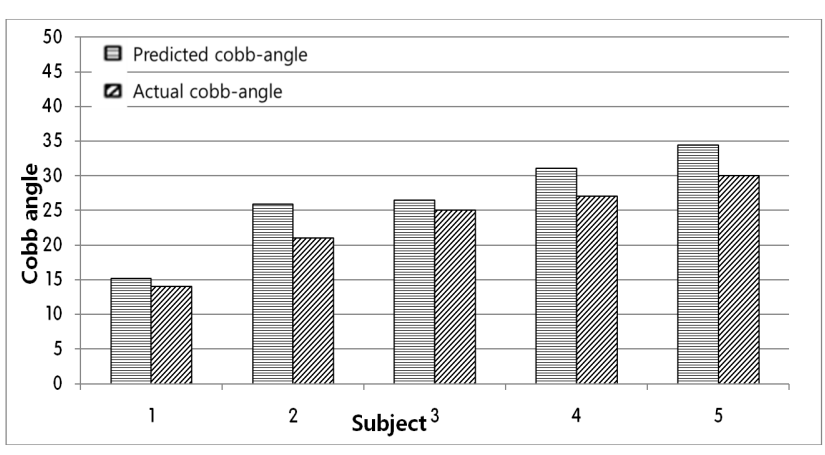

Figure 4. Result of verification. ment were used for a blind test. The measured and predicted Cobb angles are shown in Figure 4. When this model was used, the difference between the predicted values and measured values were on average $3.177^{\circ}$ with a standard deviation of $1.752^{\circ}$. Clinically, about $7^{\circ}$ of interclass error occurs during a Cobb angle measurement (Dang et al., 2005), so the result from novel prediction model can be said to have good accuracy.

\section{Conclusion}

The results of this study can be summarized as follows.

(1) 35 posture parameters of idiopathic scoliosis patients were selected based on previous studies.

(2) The 35 parameters were analyzed by ANOVA to extract parameters that were significantly different between the normal and 2 groups of scoliosis patients, which were divided based on scoliosis severity. In this analysis, 21 variables were selected.

(3) Using these 21 selected parameters, a Cobb angle prediction model was developed using multiple regression analysis. In addition data from 5 patients that were not utilized to develop the model were used for prediction model verification.

In this study, a Cobb angle prediction model was developed using posture parameters that were found to be correlated with the severity of the disease. The model had a high accuracy of $\mathrm{R}^{2}=0.9198$ and the model was verified using data from patients with an error of $5^{\circ}$. However, a number of control group data was not acquired; and the model developed in this study can only estimate the Cobb angle of scoliosis disease but cannot assess the direction or location of curvature in scoliosis patients. Therefore, if sufficient data are acquired; and information on the direction and location of curvature can be provided, effective clinical and rehabilitation strategies can be developed. Therefore, future research on the direction and location of the curvature of the spine will be needed.

\section{Conflict of Interest}

The authors have no conflicting financial or other interests 
Seo et al. Prediction of Cobb-angle for Monitoring System in Adolescent Girls with Idiopathic Scoliosis using ... Journal of Biosystems Engineering • Vol. 38, No. 1, 2013 • www.jbeng.org

\section{Acknowledgements}

This study was carried out with the support of "Cooperative Research Program for Agricultural Science \& Technology Development (Project No. 007460022012)", Rural Development Administration, Republic of Korea.

\section{References}

Byl, N. N. and J. M. Gray. 1993. Complex balance reactions in different sensory conditions: adolescents with and without idiopathic scoliosis. Journal of orthopaedic research 11(2):215-227.

Chow, D. H., M. L. Kwok, J. C. Cheng, M. L. Lao, A. D. Holmes, A. Au-Yang, F. Y. Yao and M. S. Wong. 2006. The effect of backpack weight on the standing posture and balance of schoolgirls with adolescent idiopathic scoliosis and normal controls. Gait \& Posture 24(2): 173-181.

Claus, A. P., J. A. Hides, G. L. Moseley and P. W. Hodges. 2009. Different ways to balance the spine: subtle changes in sagittal spinal curves affect regional muscle activity. Spine 34(6):E208-E214

Cobb. J. R. 1948. Outline for the study of scoliosis. In Instructional course lectures, The American academy of orthopaedic surgeons 5:261-275.

Dalleau, G., P. Leroyer, M. Beaulieu, C. Verkindt, C. H. Rivard and P. Allard. 2012. Pelvis Morphology, Trunk Posture and Standing Imbalance and Their Relations to the Cobb Angle in Moderate and Severe Untreated AIS. PLoS ONE 7(7):e36755.

Dang, N. R., M. J. Moreau, D. L. Moreau, J. K. Mahood and J. Raso. 2005. Intra-observer reproducibility and interobserver reliability of the radiographic parameters in the Spinal Deformity Study Group's AIS Radiographic Measurement Manual. Spine 30(9): 1064-1069.

Davis, R. B., S. Oonupuu, D. Tyurski and J. Gage. 1991. A gait analysis data collection and reduction. Human Movement Science 10(5):575-587.

Duval-Beaupere, G. 1971. Pathogenic relationship between scoliosis and growth. In Scoliosis and Growth. Churchill Livingstone 58-64.

Fisher, B., S. Anderson, ER. Fisher, C. Redmond, DL. Wickerham, N. Wolmark, EP. Mamounas, M. Deutsch and R. Margolese. 1991. Significance of ipsilateral breast tumour recurrence after lumpectomy. Lancet
338(8763):327-331.

Fortin, C., D. E. Feldman, F. Cheriet and H. Labelle. 2010. Validity of a quantitative clinical measurement tool of trunk posture in idiopathic scoliosis. Spine 35(19): E988-E994.

Fortin, C., D. E. Feldman, F. Cheriet and H. Labelle. 2011. Clinical methods for quantifying body segment posture: a literature review. Disability and Rehabilitation 33(5): 367-383.

Fortin, C., D. E. Feldman, F. Cheriet, D. Gravel, F. Gauthier and H. Labelle. 2012. Reliability of a quantitative clinical posture assessment tool among persons with idiopathic scoliosis. Physiotherapy 98(1):64-75.

Greendale, G. A., N. S. Nili, M. H. Huang, L. Seeger and A. S. Karlamangla. 2011. The reliability and validity of three non-radiological measures of thoracic kyphosis and their relations to the standing radiological Cobb angle. Osteoporosis International 22(6): 1897-1905.

Jaremko, J. L., P. Poncet, J. Ronsky, J. Harder, J. Dansereau, H. Labelle and R. F. Zernicke. 2002. Genetic algorithmneural network estimation of cobb angle from torso asymmetry in scoliosis. Journal of Biomechanical Engineering 124: 496-503.

Karol, L. A. 2001. Effectiveness of bracing in male patients with idiopathic scoliosis. The Spine Journal 26(18): 2001-2005.

Korovessis, P. G. and M. V. Stamatakis. 1996. Prediction of scoliotic Cobb angle with the use of the scoliometer. Spine 21(14):1661-1666.

Masso, P. D. and G. E. Gorton. 2000. Quantifying changes in standing body segment alignment following spinal instrumentation and fusion in idiopathic scoliosis using an optoelectronic using an optoelectronic measurement system. Spine 25(4): 457-462.

Nault, M. L., P. Allard, S. Hinse, R. Le Blanc, O. Caron, H. Labelle and H. Sadeghi. 2002. Relations between standing stability and body posture parameters in adolescent idiopathic scoliosis. Spine 27(17):1911-1917.

Negrini, S., G. Antonini, R. Carabalona and S. Minozzi. 2003. Physical exercises as a treatment for adolescent idiopathic scoliosis. A systematic review. Pediatric Rehabilitation 6(3-4):227-235.

Poncet, P., S. delorme, J. L. Ronsky, J. Dansereau, J. Harder and G. Clynch. 2000. Reconstruction of laser- scanned 3D torso topography and stereo-radiographical spine and rib-cage geometry in scoliosis. Computer Methods in Biomechanics and Biomedical Engineering 4(1): 59-75. 
Quervain, I. A. K., R. Müller, A. Stacoff, D. Grob and E. Stüssi. 2004. Gait analysis in patients with idiopathic scoliosis. European Spine Journal 13(5): 449-456.

Reamy, B. V. and J. B. Slakey. 2001. Adolescent idiopathic scoliosis: review and current concepts. American Family Physician 64(1): 111-116.

Sapkas, G., P. J. Papagelopoulos, K. Kateros, G. L. Koundis, P. J. Boscainos, U. I. Koukou and P. Katonis. 2003. Prediction of Cobb anlge in idiopathic adolescent scoliosis. Clinical orthopaedics and related research 411: 32-39.

Soucacos, P. N., K. Zacharis, J. Gelalis, K. Soultanis, N. Kalos, A. Beris, T. Xenakis and E.O. Johnson. 1998. Assessment of curve progression in idiopathic scoliosis. European Spine Journal 7(4):270-277.

Stokes, I. A. F., J. G. Armstrong and M. S. Moreland. 1988. Spinal deformity and back surface asymmetry in idiopathic scoliosis. Journal of orthopaedic research 6(1): 129-137.
Stokes, I. A. F. 1989. Axial rotation component of thoracic scoliosis. Journal of orthopaedic research 7(5): 702708.

Stylianides, G. A., M. Beaulieu, G. Dalleau, C. H. Rivard and P. Allard. 2012. Iliac crest orientation and geometry in able-bodied and non-treated adolescent idiopathic scoliosis girls with moderate and severe spinal deformity. European Spine Journal, 21(4):725-732.

Ylikoski, M. 1993. Spinal growth and progression of adolescent idiopathic scoliosis. European Spine Journal 1(4):236-239.

Zabjek, K. F., M. A. Leroux, C. Coillard, F. Prince and C. H. Rivard, FRCS(C), FAAOS, FACS. 2008. Postural characteristics of adolescents with idiopathic scoliosis. Journal of Pediatric Orthopaedics 28(2):218-224.

Zhu, F., W. C. Chu, G. Sun, Z. Zhu, W. Wang, J. C. Y. Cheng and Y. Qiu. 2011. Rib length asymmetry in thoracic adolescent idiopathic scoliosis: is it primary or secondary?. European Spine Journal 20(2):254-259. 